\title{
THE CLINICAL EFFECT OF HIPPOTHERAPY ON GROSS MOTOR FUNCTION OF CHILDREN WITH CEREBRAL PALSY
}

\begin{abstract}
Background: Cerebral palsy (CP) is the most common cause of physical disability affecting gross motor function (GMF) in early childhood. Hippotherapy is a treatment approach aimed at improving GMF in children with $C P$. Several systematic reviews have been published showing an improvement in Dimension E of the Gross Motor Function Measure (GMFM) after hippotherapy. However, these reviews failed to evaluate the clinical effect of hippotherapy in improving GMF in children with $C P$.

Objective: To critically appraise the evidence of hippotherapy to ascertain whether it is a clinically meaningful approach for children with $C P$.

Methodology: Five computerised bibliographic databases were searched. Predetermined inclusion and exclusion criteria were set. The PEDro scale was used to assess the quality of the studies. A revised JBI Data extraction tool was used to extract data from the selected articles. Revman $\bigcirc$ Review Manager Software was used to create forest plots for comparisons of results.
\end{abstract}

Results: All studies used the GMFM as an outcome measure for gross motor function. The added benefit of hippotherapy is a minimum $1 \%$ and a maximum $7 \%$ increase on the GMFM scores. However, all $95 \%$ confidence intervals (CI) around all the mean differences were insignificant.

Conclusion: The clinical effect of hippotherapy on the GMF of children with CP is small. Larger studies are required to provide evidence of the effect of hippotherapy within this population.

KEY WORDS: CEREBRAL PALSY, HIPPOTHERAPY, THERAPEUTIC HORSEBACK RIDING, GROSS MOTOR FUNCTION.

\section{INTRODUCTION}

Cerebral palsy (CP) is the most common cause of physical disability in early childhood (Krägeloh-Mann and Cans 2009). CP is a permanent developmental disorder, which is attributed to nonprogressive lesions that occur in the developing brain before or after birth (Rosenbaum et al 2007). The consequent impairment manifestations include scissoring gait, increased deep tendon

\section{Correspondence Author:}

Professor Quinette Louw

Division of Physiotherapy

Department of Interdisciplinary

Health Sciences

Faculty of Health Sciences

Stellenbosch University

South Africa

Email: qalouw@sun.ac.za reflexes, tremors, muscular hypertonicity and weakness (Krigger 2006). The topographic distributions are monoplegia, hemiplegia, diplegia and quadriplegia (O'Shea 2008). The classification of movement disorders assists in selecting the most appropriate management strategies for motor disturbances of children with CP.

Hippotherapy, also known as equineassisted therapy, dates back to ancient Greece, but it has only gained popularity as a treatment technique in the 1960's when it was prescribed for mental, physical and emotional issues (Meregillano 2004). It is a form of horseback riding that makes use of the smooth, rhythmical and reciprocal movement of the horse to address the physical limitations, functional impairments and disabilities of the rider (McGee and Reese 2009). Hippotherapy has been shown to improve flexibility, posture, balance and mobility of the rider (Snider et al 2007). It is proposed that when the horse moves, its centre of gravity changes three-dimensionally and its movement simulates the movement of a person's pelvis during ambulation (Drnach et al 2010). It promotes righting and balance reactions when the rider attempts to maintain the trunk and head upright during movement (Snider et al 2007). The reported benefits of hippotherapy include improved gait kinematics (Kulkarni-Lambore et al 2001) and energy expenditure (McGibbon et al 1998). Improvement in tone assists with function in sitting, standing and walking. Children that were exposed to hippotherapy showed improved selfconfidence with less fear of movement and position change.

To date four reviews related to the effect of hippotherapy have been 
published. Two of these reviews are outdated and were published in 2007 (Snider 2007, Sterba 2007). The review by Whalen and Case-Smith (2011) indicated that hippotherapy is beneficial, but failed to provide the critical appraisal of the eligible trials. A recent review by Tseng et al (2012) presented a metaanalysis, which is not appropriate due to variations in the type and duration of the interventions and study participants. This review also failed to comment on the size of the expected clinical effect on GMF which can assist clinicians in making appropriate clinical decisions.

The aim of this systematic review is therefore to critically appraise the evidence of hippotherapy or therapeutic horse riding (THR) to ascertain whether it has a clinically meaningful effect on GMF in children with CP. Therapeutic horseback riding (THR) is defined as using horseback riding treatment to improve posture, balance, and mobility while developing a therapeutic bond between the patient and horse"

\section{OBJECTIVES:}

The specific objectives of the review were to:

- Compare whether hippotherapy or THR, is more clinically meaningful in the short as well as long term (longer than 12 months), in improving gross motor function compared to usual therapies,

- Describe the type and dosage of usual therapies employed in eligible studies,

- Describe the type and dosage of hippotherapy employed in eligible studies,

- Describe which type of gross motor outcome measures were used in eligible studies.

\section{METHODOLOGY}

The eligibility criteria of studies were as follows:

\section{Types of Studies:}

- Randomised controlled trials (RCTs), non-randomised controlled trials and prospective case series were considered for inclusion in this review.

- Only articles published in the English language were considered for eligibility.
- All articles published from inception of the databases until March 2012 were considered.

\section{Types of Participants:}

- Male and female children aged between 2-12 years old. Children under the age of 2 years are not accepted for hippotherapy. (http://www.3gaits.org)

13- to 17 year olds are regarded as adolescence.

- Diagnosed with CP.

\section{Types of Interventions:}

- Hippotherapy or THR combined with usual therapies.

\section{Types of Comparisons:}

- Usual therapies include any therapy that the child routinely participates in, but not only confined to physiotherapy, occupational therapy, special education or speech therapy.

Types of Outcome Measures:

- Gross Motor Function Measure (GMFM) 66 and GMFM 88.

\section{SEARCH STRATEGY}

Five computerised bibliographical databases accessed through the Stellenbosch University library services were searched including; CINAHL, Cochrane Library, Pubmed, PEDro and Science Direct. Database specific search strategies were developed for each database.

The following key search terms were used: cerebral palsy, equine-assisted therapy, gross motor function, hippotherapy and therapeutic horseback riding.

\section{METHODOLOGICAL APPRAISAL}

The PEDro scale was used to critically appraise the internal validity of the studies (Pearson et al 2009). Two researchers independently appraised full text of articles, and a third reviewer was consulted to reach consensus about disagreements.

\section{DATA EXTRACTION METHOD}

Databases were searched independently by one researcher. Databases were then searched a second time by a different researcher, thereby automatically cross checking one another's appointed database. The supervisor was contacted if no consensus was found amongst researchers. Pearling was also administered by both researchers.

Two researchers (KL and NN) independently did the data extraction from an article. Should the researchers fail to obtain the necessary information from an article, the authors were contacted to complete the data extraction. After the data extraction the two researchers compared their data. If consensus about the data extraction was not reached, the two researchers presented their differences to a third researcher. The third researcher arbitrated and if consensus could still not be reached among the three researchers, the supervisor was contacted.

A standard JBI Data Extraction form (Sussman \& Aiona, 2004); (Bertoti, 1988) (Sterba, 2007); (Whalen \& CaseSmith, 2011) was used to extract the following; study citation, study method, participants, interventions (treatment and control group), outcome measures, results, clinical status post-intervention and clinical implications.

\section{DATA ANALYSIS}

Studies identified as having comparable data were combined using Revman ${ }^{\odot}$ Review Manager software to calculate mean differences and $95 \% \mathrm{CI}$ when the mean and standard deviations for the specific study was available (RevMan ${ }^{\odot}$ information management system 2011). The mean and $95 \%$ CI were graphically presented using forest plots. Due to heterogeneity meta-analysis was not possible. Studies which did not provide mean and standard deviations could not be included in the forest plots but were included by describing their results in a narrative format.

\section{RESULTS}

A total of 74 hits were obtained. Of these, 22 abstracts were reviewed and 12 full-text articles were subsequently considered as being potentially eligible for use in this systematic review. Of these 12 full-text articles, 6 articles did not meet the inclusion criteria. Therefore, 6 eligible full-text articles were included in this systematic review (Figure 1). 
None of the studies considered and reported on the long term effect of hippotherapy on children with $\mathrm{CP}$.

\section{METHODOLOGICAL APPRAISAL}

The methodological quality of the final six articles was assessed using the eleven-item PEDro scale and the articles scored between $4 / 11$ and $7 / 11$, with an average score of $6.33 / 11(57.57 \%)$. The PEDro scale, based on the Delphi list, consists of eleven criteria, measuring a study's internal validity (criteria 2-9); external validity (criteria 1), as well as the statistical accuracy for interpretation purposes (criteria 10-11) (Pearson, Field and Jordan, 2009). Table 1 provides an overview of the PEDro scores. During the methodological appraisal of the final articles, it was noticed that criteria 5 (blinding of all subjects) and 6 (blinding of the therapists) was impossible for this intervention.

Table 1: Pedro Scores

\begin{tabular}{|c|c|c|c|c|c|c|}
\hline PEDro criteria & $\begin{array}{l}\text { Casady } \\
\text { \& Nichols- } \\
\text { Larsen } 2004\end{array}$ & $\begin{array}{l}\text { Cherng } \\
\text { et al. } \\
2004\end{array}$ & $\begin{array}{l}\text { Davis } \\
\text { et al. } \\
2009\end{array}$ & $\begin{array}{l}\text { Kwon } \\
\text { et al. } \\
2011\end{array}$ & $\begin{array}{l}\text { MacKinnon } \\
\text { et al. } \\
1995\end{array}$ & $\begin{array}{l}\text { McGibbon } \\
\text { et al. } \\
1998\end{array}$ \\
\hline 1. Eligibility criteria were specified & $\checkmark$ & $\checkmark$ & $\checkmark$ & $\checkmark$ & $\checkmark$ & $\checkmark$ \\
\hline $\begin{array}{l}\text { 2. Subjects were randomly allocated to } \\
\text { groups (in a crossover study, subjects } \\
\text { were randomly allocated an order in } \\
\text { which treatments were received) }\end{array}$ & $x$ & $\checkmark$ & $\checkmark$ & $x$ & $\checkmark$ & $x$ \\
\hline 3. Allocation was concealed & $x$ & $x$ & $\checkmark$ & $x$ & $x$ & $x$ \\
\hline $\begin{array}{l}\text { 4. The groups were similar at baseline } \\
\text { regarding the most important } \\
\text { prognostic indicators }\end{array}$ & $\checkmark$ & $\checkmark$ & $\checkmark$ & $\checkmark$ & $\checkmark$ & $\checkmark$ \\
\hline 5. There was blinding of all subjects & $x$ & $x$ & $x$ & $x$ & $x$ & $x$ \\
\hline $\begin{array}{l}\text { 6. There was blinding of all therapists } \\
\text { who administered the therapy }\end{array}$ & $x$ & $x$ & $x$ & $x$ & $x$ & $x$ \\
\hline $\begin{array}{l}\text { 7. There was blinding of all assessors } \\
\text { who measured at least one key } \\
\text { outcome }\end{array}$ & $x$ & $\checkmark$ & $x$ & $\checkmark$ & $\checkmark$ & $x$ \\
\hline $\begin{array}{l}\text { 8. Measures of at least one key } \\
\text { outcome were obtained from more } \\
\text { than } 85 \% \text { of the subjects initially } \\
\text { allocated to groups }\end{array}$ & $\checkmark$ & $x$ & $x$ & $\checkmark$ & $\checkmark$ & $\checkmark$ \\
\hline $\begin{array}{l}\text { 9. All subjects for whom outcome } \\
\text { measures were available received } \\
\text { the treatment or control condition as } \\
\text { allocated or, where this was } \\
\text { not the case, data for at least one } \\
\text { key outcome was analysed by } \\
\text { "intention to treat" }\end{array}$ & $\checkmark$ & $\checkmark$ & $\checkmark$ & $\checkmark$ & $\checkmark$ & $\checkmark$ \\
\hline $\begin{array}{l}\text { 10. The results of between-group } \\
\text { statistical comparisons are reported } \\
\text { for at least one key outcome }\end{array}$ & $\checkmark$ & $\checkmark$ & $\checkmark$ & $\checkmark$ & $x$ & $x$ \\
\hline $\begin{array}{l}\text { 11. The study provides both point } \\
\text { measures and measures of variability } \\
\text { for at least one key outcome }\end{array}$ & $\checkmark$ & $\checkmark$ & $\checkmark$ & $\checkmark$ & $\checkmark$ & $x$ \\
\hline Total score & $5 / 10$ & $6 / 10$ & $6 / 10$ & $6 / 10$ & $6 / 10$ & $3 / 10$ \\
\hline
\end{tabular}

\begin{tabular}{|l|l|c|l|}
\hline \multicolumn{4}{|c|}{ Key: } \\
\hline$\checkmark$ & YES & $\times$ & NO \\
\hline
\end{tabular}


Table 2: Study sample description

\begin{tabular}{|c|c|c|c|c|c|c|c|}
\hline & $\begin{array}{c}\text { Casady \& } \\
\text { Nichols-Larsen } \\
2004\end{array}$ & $\begin{array}{l}\text { Cherng } \\
\text { et al. } \\
2004\end{array}$ & $\begin{array}{l}\text { Davis } \\
\text { et al. } \\
2009\end{array}$ & $\begin{array}{l}\text { Kwon } \\
\text { et al. } \\
2011\end{array}$ & $\begin{array}{c}\text { MacKinnon } \\
\text { et al. } \\
1995\end{array}$ & $\begin{array}{l}\text { McGibbon } \\
\text { et al. } \\
1998\end{array}$ \\
\hline \multicolumn{2}{|c|}{ Type of study } & $\begin{array}{c}\text { Quasi- } \\
\text { experimental } \\
\text { self-control }\end{array}$ & $\begin{array}{c}\text { Randomised } \\
\text { cross over }\end{array}$ & $\begin{array}{l}\text { Randomised } \\
\text { cross over }\end{array}$ & $\begin{array}{l}\text { Non- } \\
\text { randomized } \\
\text { prospective }\end{array}$ & $\begin{array}{l}\text { Randomised } \\
\text { control trail }\end{array}$ & $\begin{array}{l}\text { Repeated } \\
\text { measures self- }\end{array}$ \\
\hline \multirow{2}{*}{ Sample size } & $\begin{array}{l}\text { Intervention } \\
\text { Group }\end{array}$ & 10 & 35 & 9 & 10 & 5 & 16 \\
\hline & Control Group & 10 & 37 & 5 & 9 & 5 & 16 \\
\hline \multirow{2}{*}{ Gender } & $\begin{array}{l}\text { Intervention } \\
\text { Group }\end{array}$ & $\begin{array}{c}\text { Male }=6 \\
\text { Female }=4\end{array}$ & $\begin{array}{c}\text { Male=17 } \\
\text { Female=18 }\end{array}$ & $\begin{array}{c}\text { Male=7 } \\
\text { Female=2 }\end{array}$ & $\begin{array}{c}\text { Male=3 } \\
\text { Female=7 }\end{array}$ & $\begin{array}{c}\text { Male=3 } \\
\text { Female=2 }\end{array}$ & $\begin{array}{l}\text { Male }=11 \\
\text { Female=5 }\end{array}$ \\
\hline & Control Group & $\begin{array}{c}\text { Male }=6 \\
\text { Female }=4\end{array}$ & $\begin{array}{c}\text { Male }=20 \\
\text { Female }=17\end{array}$ & $\begin{array}{c}\text { Male=1 } \\
\text { Female=4 }\end{array}$ & $\begin{array}{c}\text { Male }=6 \\
\text { Female=3 }\end{array}$ & $\begin{array}{c}\text { Male=3 } \\
\text { Female=2 }\end{array}$ & $\begin{array}{l}\text { Male }=10 \\
\text { Female }=6\end{array}$ \\
\hline \multirow{2}{*}{$\begin{array}{l}\text { Age } \\
\text { (in years) }\end{array}$} & $\begin{array}{l}\text { Intervention } \\
\text { Group }\end{array}$ & $\begin{array}{c}\text { Mean (SD): } \\
4.1+1.7\end{array}$ & $\begin{array}{l}\text { Mean (SD): } \\
7.4 \pm 2.5\end{array}$ & $\begin{array}{l}\text { Mean (SD): } \\
7.69 \pm 2.68\end{array}$ & $\begin{array}{c}\text { Mean (SD): } \\
7 \pm 2.2\end{array}$ & $\begin{array}{c}\text { Mean: } \\
9.6\end{array}$ & $\begin{array}{c}\text { Mean (SD): } \\
6.4 \pm 1.7\end{array}$ \\
\hline & Control Group & $\begin{array}{c}\text { Mean (SD): } \\
4.1+1.7\end{array}$ & $\begin{array}{l}\text { Mean (SD): } \\
7.9 \pm 2.4\end{array}$ & $\begin{array}{l}\text { Mean (SD): } \\
7.75 \pm 1.60\end{array}$ & $\begin{array}{l}\text { Mean (SD): } \\
5.85 \pm 1.7\end{array}$ & $\begin{array}{l}\text { Mean: } \\
9.6\end{array}$ & $\begin{array}{c}\text { Mean (SD): } \\
6.1 \pm 1.7\end{array}$ \\
\hline \multicolumn{2}{|c|}{$\begin{array}{l}\text { Country where study was } \\
\text { performed }\end{array}$} & $\begin{array}{l}\text { United states } \\
\text { of America }\end{array}$ & Taiwan & Australia & Korea & $\begin{array}{l}\text { United } \\
\text { Kingdom }\end{array}$ & $\begin{array}{l}\text { United States } \\
\text { of America }\end{array}$ \\
\hline
\end{tabular}

SD: Standard deviation

\section{STUDY SAMPLE DESCRIPTION}

A summary of the sample descriptions for each study is shown in Table 2.

\section{DESCRIPTION OF INTERVENTIONS}

Studies either used hippotherapy or THR as their intervention; both were aimed at improving GMF by adapting the position or movements of the child while on the horse. Participants were encouraged to touch various parts of the horse or perform different tasks while maintaining balance and posture, depending on the goal of the treatment session. All of the studies allowed participants in the intervention group to continue with their regular treatment which varied between the studies (Table 3 ). The control groups were instructed to continue with their normal therapeutic routine as described in Table 3, except Kwon et al (2011) who received conventional physiotherapy consisting of neurodevelopmental therapy.

\section{DESCRIPTION OF OUTCOME MEA- SURES}

All studies used a version of the GMFM in order to assess gross motor function (Table 4). The original GMFM-88, or parts thereof, was used by all studies except Davis et al (2009), who used the more recent and compact GMFM-66. Kwon et al (2011), made use of both the GMFM-66 and GMFM-88.

\section{EFFECT OF HIPPOTHERAPY COM- PARED TO USUAL THERAPIES}

The effects of the interventions investigated are described in the following figures and narration, addressing each outcome separately.

GMFM - 88: Dimension A (lying and rolling), Dimension B (sitting) and Dimension $C$ (crawling and kneeling)

Casady and Nichols-Larsen (2004), and Cherng et al (2004), reported that there was no statistical significant difference in Dimensions A and C. Three of the articles also reported an insignificant statistical difference for Dimension B (Casady and Nichols-Larsen, 2004, Cherng et al 2004 and MacKinnon et al 1995). Unfortunately the data was not available to calculate forest plots.

\section{GMFM - 88: Dimension D (standing)}

The mean difference reported in three studies favoured the hippotherapy group and ranged from 3.2 to 4.1 for the GMFM 88: Dimension D (Figure 2). The $95 \%$ confidence intervals were insignificant.

\section{GMFM - 88: Dimension E (walking, running and jumping)}

The mean difference of the four studies reporting on Dimension $\mathrm{E}$ illustrated positive mean differences for hippotherapy ranging from 1.3 to 7.7 (Figure 2). The 95\% confidence intervals were insignificant. 
Table 3: Description of interventions

\begin{tabular}{|c|c|c|c|c|c|c|c|}
\hline & & $\begin{array}{l}\text { Casady \& } \\
\text { Nichols- } \\
\text { Larsen } \\
2004\end{array}$ & $\begin{array}{l}\text { Cherng } \\
\text { et. al. } \\
2004\end{array}$ & $\begin{array}{l}\text { Davis } \\
\text { et. al. } \\
2009\end{array}$ & $\begin{array}{l}\text { Kwon } \\
\text { et. al. } \\
2011\end{array}$ & $\begin{array}{c}\text { MacKinnon } \\
\text { et al. } \\
1995\end{array}$ & $\begin{array}{l}\text { McGibbon } \\
\text { et. al. } \\
1998\end{array}$ \\
\hline \multirow[t]{2}{*}{ 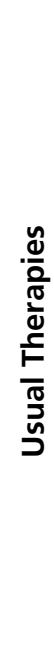 } & 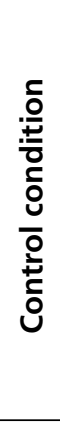 & $\begin{array}{l}\text { - Continue } \\
\text { therapies at } \\
\text { school and } \\
\text { clinics. }\end{array}$ & $\begin{array}{l}\text { - Passive ROM } \\
\text { exercises, } \\
\text { positioning, } \\
\text { balance } \\
\text { training, } \\
\text { functional } \\
\text { training and } \\
\text { Neuro- } \\
\text { developmental } \\
\text { training }\end{array}$ & $\begin{array}{l}\text { - Continued } \\
\text { with normal } \\
\text { or daily } \\
\text { routine } \\
\text { including } \\
\text { physiotherapy. } \\
\text { (techniques } \\
\text { not specified) }\end{array}$ & $\begin{array}{l}\text { - Neuro- } \\
\text { developmental } \\
\text { therapy }\end{array}$ & $\begin{array}{l}\text { - Not specified } \\
\text { - No attempts } \\
\text { were made to } \\
\text { stop routine } \\
\text { therapies or } \\
\text { activities in } \\
\text { which the } \\
\text { children were } \\
\text { engaged }\end{array}$ & $\begin{array}{l}\text { - Continued } \\
\text { with } \\
\text { normal daily } \\
\text { routine. }\end{array}$ \\
\hline & $\begin{array}{l}\frac{1}{0} \\
\frac{0}{\pi} \\
\frac{0}{5} \\
0\end{array}$ & - 10 weeks & $\begin{array}{l}16 \text { weeks } \\
\text { control }\end{array}$ & - 10 weeks & $\begin{array}{l}\text { - } 8 \text { weeks } \\
\text { - Twice a week } \\
\text { for } 30 \text { minutes }\end{array}$ & - 26 weeks & - 8 weeks \\
\hline \multirow[t]{2}{*}{ 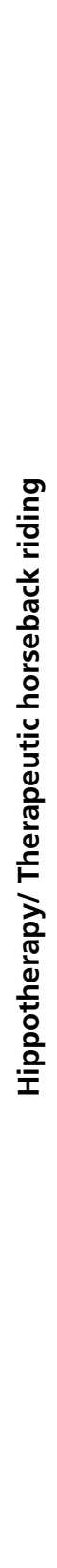 } & 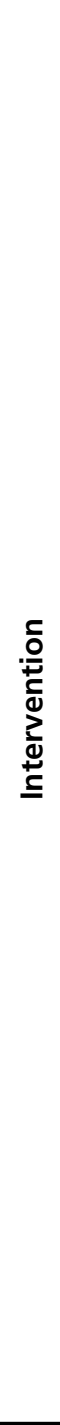 & $\begin{array}{l}\text { - Hippo- } \\
\text { therapy } \\
\text { - Subjects } \\
\text { were } \\
\text { encouraged } \\
\text { to maintain } \\
\text { postural } \\
\text { alignment } \\
\text { with } \\
\text { symmetry of } \\
\text { head, trunk } \\
\text { and lower } \\
\text { extremities. } \\
\text { - Sit indepen- } \\
\text { dently on } \\
\text { horse with } \\
\text { little or no } \\
\text { assistance. } \\
\text { - Continued } \\
\text { with other } \\
\text { therapies } \\
\text { such as } \\
\text { physio- } \\
\text { therapy, OT } \\
\text { or speech } \\
\text { therapy if } \\
\text { applicable }\end{array}$ & $\begin{array}{l}\text { - Therapeutic } \\
\text { horseback } \\
\text { riding to } \\
\text { maintain good } \\
\text { posture. } \\
\text { - Movement of } \\
\text { arms including } \\
\text { elevation and } \\
\text { abduction of } \\
\text { shoulder. } \\
\text { - Trunk flexion, } \\
\text { rotational } \\
\text { movement } \\
\text { while on the } \\
\text { horse. } \\
\text { - Touching } \\
\text { various } \\
\text { parts of the } \\
\text { horse's body. } \\
\text { - Regular } \\
\text { treatment } \\
\text { continued (eg } \\
\text { physiotherapy, } \\
\text { occupational } \\
\text { therapy } \\
\text { and special } \\
\text { education) }\end{array}$ & $\begin{array}{l}\text { - THR with } \\
\text { activities } \\
\text { designed to } \\
\text { emphasize } \\
\text { movement in } \\
\text { a forward and } \\
\text { upward } \\
\text { reaching } \\
\text { direction. } \\
\text { - Continued } \\
\text { with } \\
\text { normal or } \\
\text { daily routine } \\
\text { including } \\
\text { physiotherapy. }\end{array}$ & $\begin{array}{l}\text { - Hippotherapy } \\
\text { for relaxation } \\
\text { at start of } \\
\text { each session } \\
\text { feeling the } \\
\text { gentle, } \\
\text { rhythmical } \\
\text { movement of } \\
\text { the horse. } \\
\text { - Sustaining } \\
\text { optimal } \\
\text { postural } \\
\text { alignment } \\
\text { - Active } \\
\text { exercises for } \\
\text { stretching, } \\
\text { strengthening, } \\
\text { and dynamic } \\
\text { weight shift. } \\
\text { - Conventional } \\
\text { physiotherapy }\end{array}$ & $\begin{array}{l}\text { - THR } \\
\text { - No attempt } \\
\text { was made to } \\
\text { stop routine } \\
\text { therapies }\end{array}$ & $\begin{array}{l}\text { - Hippo- } \\
\text { therapy for } \\
\text { relaxation } \\
\text { at start of } \\
\text { each ses- } \\
\text { sion feeling } \\
\text { the gentle, } \\
\text { rhythmical } \\
\text { movement } \\
\text { of the horse. } \\
\text { Forward } \\
\text { sitting } \\
\text { - Backward } \\
\text { sitting (for } \\
\text { thoracic } \\
\text { extension } \\
\text { and a more } \\
\text { anterior } \\
\text { pelvic tilt). } \\
\text { Active } \\
\text { exercises for } \\
\text { stretching, } \\
\text { strengthen- } \\
\text { ing, and } \\
\text { dynamic } \\
\text { weight shift. } \\
\text { - One child } \\
\text { was receiv- } \\
\text { ing physical } \\
\text { therapy. No } \\
\text { other treat- } \\
\text { ments were } \\
\text { initiated or } \\
\text { discontinued }\end{array}$ \\
\hline & $\begin{array}{l}\frac{2}{0} \\
\stackrel{0}{+} \\
\frac{0}{3} \\
0\end{array}$ & $\begin{array}{l}10 \text { weeks, } \\
\text { once a } \\
\text { week for } \\
45 \text { minutes. }\end{array}$ & $\begin{array}{l}\text { - } 16 \text { weeks of } \\
\text { THR twice a } \\
\text { week, } \\
40 \text { minute } \\
\text { sessions. }\end{array}$ & $\begin{array}{l}\text { - } 10 \text { week pro- } \\
\text { gram, once a } \\
\text { week for } 30-40 \\
\text { minutes. }\end{array}$ & $\begin{array}{l}\text { - } 8 \text { weeks, twice } \\
\text { a week for } 30 \\
\text { minutes. }\end{array}$ & $\begin{array}{l}\text { - } 26 \text { weeks of } \\
\text { THR, once a } \\
\text { week for an } \\
\text { hour }\end{array}$ & $\begin{array}{l}\text { - } 8 \text { weeks, } \\
\text { twice } \\
\text { weekly for } \\
30 \text { minutes. }\end{array}$ \\
\hline
\end{tabular}


Table 4: Description of outcome measures used to measure gross motor function, as well as follow-up measurement intervals

\begin{tabular}{|c|c|c|}
\hline & Outcome Measure (OM) & Assessment Intervals \\
\hline $\begin{array}{l}\text { Casady \& } \\
\text { Nichols-Larsen } \\
2004\end{array}$ & 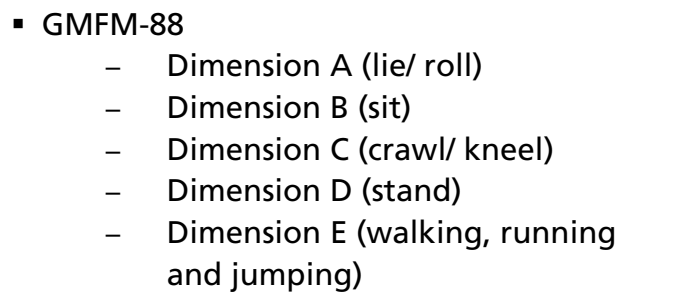 & $\begin{array}{l}\text { - } \text { Pre-test } 1 \\
\text { - } \text { Pre-test } 2 \\
\text { - } \text { Post-test } 1 \\
\text { - } \text { Post-test } 2 \\
10 \text { week intervals }\end{array}$ \\
\hline $\begin{array}{l}\text { Cherng et al. } \\
2004\end{array}$ & 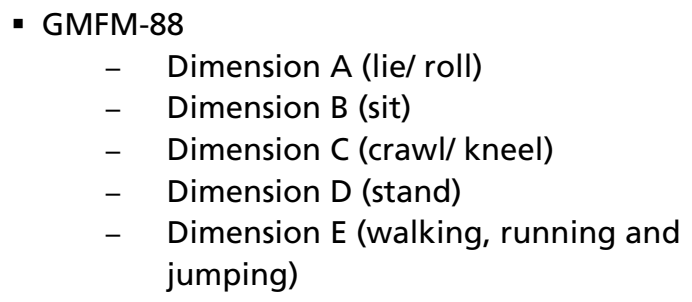 & $\begin{array}{l}\text { - Baseline } \\
\text { - End of } 16 \text { weeks cross over } \\
\text { - End of } 2^{\text {nd }} \text { period of } 16 \text { weeks }\end{array}$ \\
\hline $\begin{array}{c}\text { Davis et al. } \\
2009\end{array}$ & - GMFM-66 & $\begin{array}{l}\text { - Baseline } \\
\text { - End of } 10 \text { week intervention period }\end{array}$ \\
\hline $\begin{array}{l}\text { Kwon et al. } \\
2011\end{array}$ & 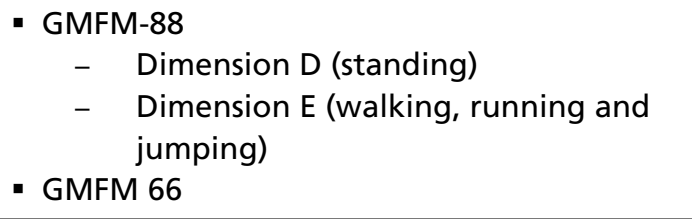 & $\begin{array}{l}\text { - Baseline } \\
\text { - End of } 8 \text { week intervention period }\end{array}$ \\
\hline $\begin{array}{l}\text { MacKinnon } \\
\text { et al. } 1995\end{array}$ & $\begin{array}{l}\text { - GMFM-88 } \\
\begin{aligned}- & \text { Dimension B (sit) } \\
- & \text { Dimension D (stand) } \\
- & \text { Dimension E (walking, running and } \\
& \text { jumping) }\end{aligned}\end{array}$ & $\begin{array}{l}\text { - Baseline } \\
\text { - End of } 26 \text { weeks }\end{array}$ \\
\hline $\begin{array}{l}\text { McGibbon et al. } \\
1998\end{array}$ & $\begin{array}{l}\text { - GMFM-88 } \\
\begin{array}{l}-\quad \text { Dimension E (walking, running, } \\
\text { jumping) }\end{array}\end{array}$ & $\begin{array}{l}\text { - Baseline } \\
\text { - Baseline after } 8 \text { weeks } \\
\text { - End of } 8 \text { weeks post equine assisted therapy }\end{array}$ \\
\hline
\end{tabular}

\section{GMFM-88: Total Score}

The mean difference of the studies for which it was possible to calculate the mean difference of the total GMFM88 score was 0.96 to 3.68 in favour of hippotherapy (Figure 2).

\section{GMFM-66: Total Score}

The mean difference for the GMFM66 total score between Davis et al (2009) and Kwon et al (2011) indicated equivocal findings since the mean difference of Davis et al favoured usual therapies and Kwon et al (2011) found that hippotherapy in combination of usual therapy was superior to usual therapy alone (Figure 2).

\section{DISCUSSION}

This review endeavoured to determine the clinical effect of hippotherapy com- bined with usual therapies on gross motor function in comparison to usual therapies in the short as well as the long term. This study calculated mean difference and CI, which is the most commonly used measure of clinical effect (Ranstam 2012). Although statistical significance is often reported, clinicians are also guided by the magnitude of the effect of an intervention to ascertain if the therapy will have clinically meaningful effect. The main finding of this review is that hippotherapy does not result in a clinically meaningful effect compared to usual therapies in improving gross motor function in children with CP.

The hippotherapy sessions varied between thirty minutes and an hour and were performed once or twice a week; this indicates that duration and frequency did not influence the effect of the intervention. However, the main finding of this review is that the clinically meaningful effect of hippotherapy in the short term is small. The tendency that hippotherapy results in positive effects on gross motor function, were also indicted in a recent systematic review by Whalen and Case-Smith (2011), as well as a review by Sterba (2007). These reviews only reported statistical significance; the lack of clinically meaningful effect is a key shortcoming which is required by clinicians to assist them in deciding whether hippotherapy will yield added benefit. None of the studies considered and reported on the long term effect of hippotherapy on children with CP.

The current evidence base for the effect of hippotherapy on gross motor function is limited. Although only six studies were included in this review, 


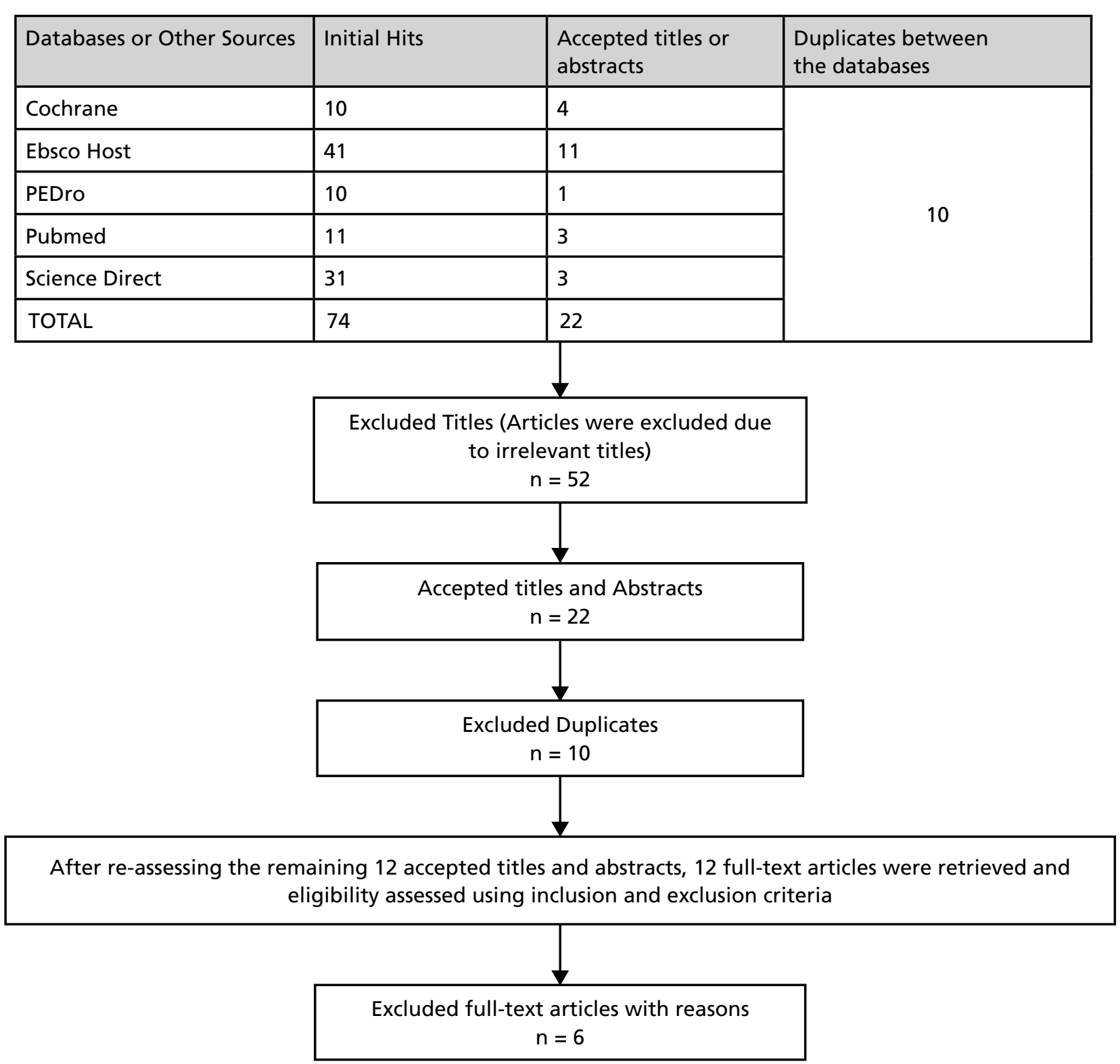

Eligible articles for this systematic review

$$
\mathrm{n}=6
$$

\section{Figure 1: Results of search strategy}

it should be noted that the search was limited to five databases and only papers published in the English language were eligible. This review was limited to children between 2-12 years of age and all types of CP were included in order to broaden the search. An understanding of the effect of hippotherapy is further complicated by the variation in study designs. This review's search strategy results indicated that only one randomised controlled clinical trial was eligible. A lack of randomisation is one key threat to internal validity which transpired after critical appraisal of the eligible studies. Randomised controlled trials remain the mainstay of the highest level of primary research evidence due to the robust internal validity of a well conducted trial. Researchers in this field should conduct more randomised controlled trials as they are essential in guiding evidence based decision making for clinicians.

Several limitations in the included articles were identified. Five of the six studies used in this review included very small samples and no justification for the number of subjects were included. This trend could be due to the high cost of therapy or strict inclusion criteria. The small sample sizes increases the risk of bias and were most likely of insufficient power to detect significant changes in gross motor function. Generalisation of the findings of individual studies may be limited as these relatively small samples are not representative of the $\mathrm{CP}$ population, therefore clinicians should 


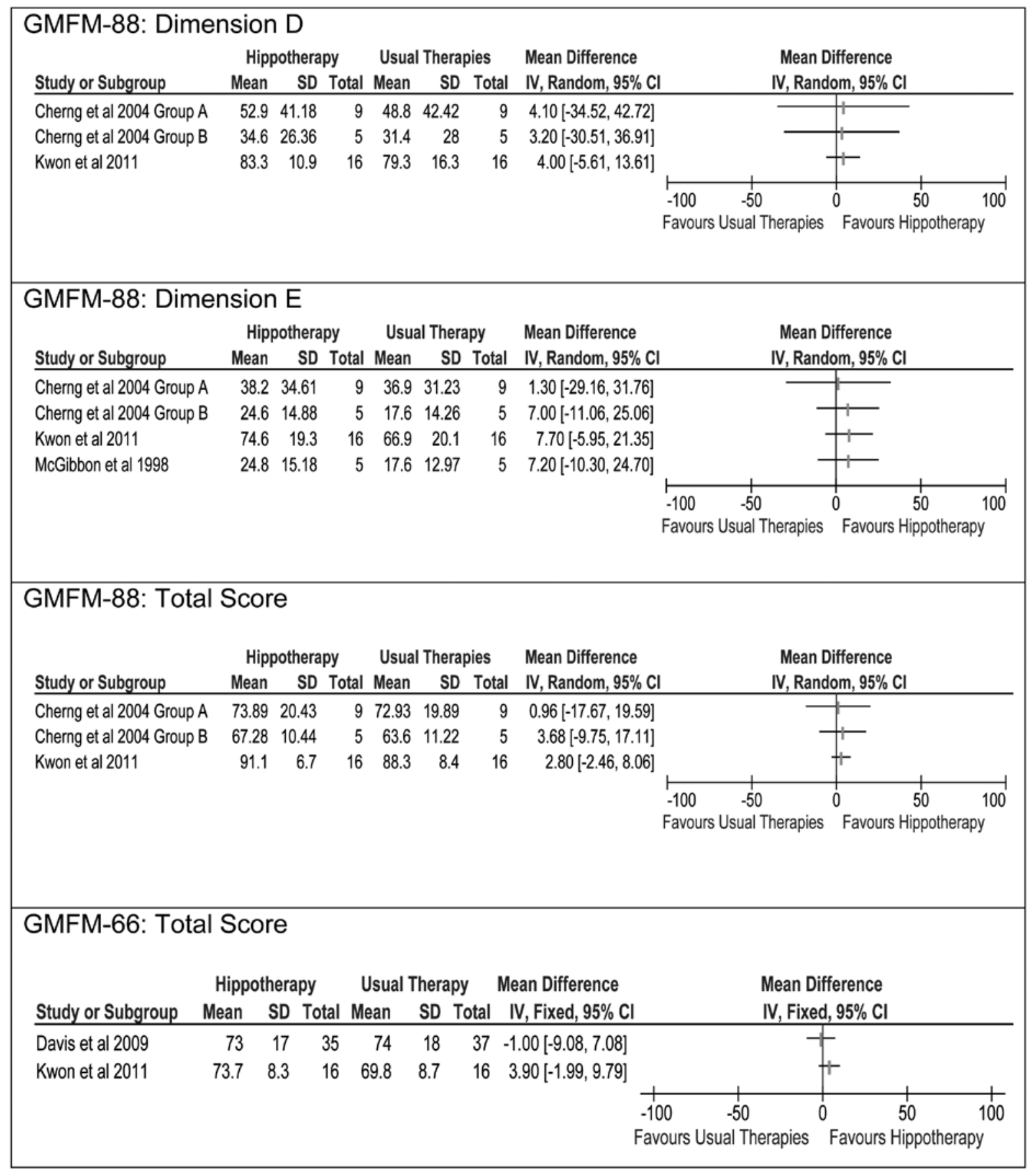

\section{Figure 2: Forest plots}

make use of meta-analyses to appraise the evidence.

This review highlights the variability in the current evidence base for the effect of hippotherapy. Variation in inclusion criteria of subjects is one aspect which should be standardised in future studies. For instance, the inclusion criteria described by Kwon et al (2011), required participants to be Level I or II on the gross motor function classification scale whilst participants in the study done by Cherng et al (2004), varied in function from crawling on belly to walking independently. These baseline levels determine the potential for improvement. Since subjects could already walk in some of the eligible studies, it is expected that there will be no difference in Dimension D of the GMFM-88 which measures standing ability. This aspect of statistical regression has been explained by Cherng et al (2004), who concluded that Dimension A and B of the GMFM88 was statistically insignificant due to the ceiling effect. Future studies should therefore attempt to standardise the eligible criteria, which will facilitate comparison across groups.

The studies included in this review are of moderate methodological quality as well as the moderate to high level of evidence on the hierarchy of evidence. The hierarchy of evidence is a useful tool that can be used to appraise the validity and reliability of a particular study.

Studies reported the duration and frequency of the intervention as well as what the intervention entailed, which 
can give therapists a guideline for clinical practice or it can be used for comparisons to either existing or future studies. Cherng et al (2004), Casady and Nichols-Larsen (2004) and Davis et al (2009) conducted a within participant repeated measure which allows more accurate comparison between baseline and post-intervention comparisons. Further research should be done using self-controls, as children with the same diagnosis of CP have different clinical presentations.

Heterogeneity in the control condition of the eligible studies was notable as none of the studies could delineate the effect of hippotherapy from a specific type of therapy. This could be because it would be unethical to request that subject cease usual therapy. Due to these variations, statistical pooling was impossible and the clinical effect of each individual study was appraised by calculating the mean difference and CI. A meta-analysis is one approach which can be applied to combine the findings of two or more primary studies and therefore increase the statistical power of primary studies. Unfortunately it was not possible to conduct a meta-analysis due to heterogeneity in study variables and characteristics.

It is also plausible that other associated benefits such as emotional, cognitive, social, communication and increased self-esteem may influence the decision to include hippotherapy in the management of children with CP (Meregillano 2004). However, the evidence which was systematically appraised in this study indicates that hippotherapy in conjunction with usual therapies does not result in a clinically meaningful effect compared to usual therapies alone.

\section{CLINICAL IMPLICATIONS AND DIREC- TIONS FOR FUTURE RESEARCH}

Hippotherapy in combination with usual therapies is not superior to usual therapy alone, and it does not have a clinically meaningful effect:

- The clinical effect of hippotherapy is negligible as a minimum of $1 \%$ and a maximum $7 \%$ increase is expected on the GMFM scores.

- The estimated clinical effect in the population is insignificant. Therefore clinicians need to consider factors such as financial cost and potential harm (e.g. falling off horse) when deciding whether it is clinically worthwhile to include hippotherapy in the rehabilitation program of children with CP.

Future, pragmatic, well conducted trials are required to provide conclusive evidence pertaining to the clinical effect of hippotherapy in children with CP.

\section{REFERENCES}

Anita C All, Gary L Loving \& Laura Lee Crane. 1999. Animals, horseback riding, and implications for rehabilitation therapy. Journal of Rehabilitation, 65(3): 49-57.

Bertoti, D.B. 1988. Effect of therapeutic horseback riding on posture in children with cerebral palsy. Physical Therapy, 68(10): 1505-1512. Casady R, Nichols-Larsen D 2004 The effect of hippotherapy on ten children with cerebral palsy. Pediatric Physical Therapy 16(3): 165-172

Cherng R, Liao H, Leung H, Hwang A 2004 The effectiveness of therapeutic horseback riding in children with spastic cerebral palsy. Adapted Physical Activity Quarterly 21(2): 103-121

Davis E 2009. A randomized controlled trial of the impact of therapeutic horse riding on the quality of life, health, and function of children with cerebral palsy. Developmental Medicine and Child Neurology 51(2): 111-119

Drnach M, O’Brien PA, Kreger A 2010 The effects of a 5-week therapeutic horseback riding program on gross motor function in a child with cerebral palsy: A case study. Journal of Alternative and Complementary Medicine 16(9): 1003-1006

Krägeloh-Mann I, Cans C 2009 Cerebral palsy update. Brain and Development 31(7): 537-544

Krigger KW, 2006 Cerebral palsy: An overview. American Family Physician 73(1): 91-100

Kulkarni-Lambore S, McGuigan A, Narula N, Sepelak K 2001 Kinematic gait analysis of an individual with cerebral palsy before and after hippotherapy. Physical Therapy 81 (5): A40

Kwon J, Chang HJ, Lee JY, Ha Y, Lee PK, Kim Y 2011 Effects of hippotherapy on gait parameters in children with bilateral spastic cerebral palsy. Archives of Physical Medicine and Rehabilitation 92(5): 774-779

MacKinnon JR, Noh S, Lariviere J, MacPhil A, Allan DE, Laliberte D 1995 A study of therapeutic effects of horseback riding for children with cerebral palsy. Physical \& Occupational Therapy in Pediatrics 15(1): 17-34
McGee MC, Reese NB 2009 Immediate effects of a hippotherapy session on gait parameters in children with spastic cerebral palsy. Pediatric Physical Therapy 21(2): 212-218

McGibbon NH, Andrade C, Widener G, Cintas HL 1998 Effect of an equine-movement therapy program on gait, energy expenditure, and motor function in children with spastic cerebral palsy: A pilot study. Developmental Medicine \& Child Neurology 40(11): 754-762

Meregillano G 2004 Hippotherapy. Physical Medicine and Rehabilitation Clinics of North America 15(4): $843-854$

O'Shea M 2008 Cerebral palsy. Seminars in Perinatology 32(1): 35-41

Pearson A, Field J, Jordan Z Appendix 3: Data extraction tools, in evidence-based clinical practice in nursing and health care: Assimilating research, experience and expertise. [Online]. Available: http://onlinelibrary.wiley.com/doi/10.1002/ 9781444316544.app3/pdf [2012, January 25]

Ranstam J 2012. Why the P-value culture is bad and confidence intervals a better alternative. Osteoarthritis and Cartilage Editorial 1-4

RevMan( information management system. [Online].

Available: http://www.cc-ims.net/RevMan [2012, January 18]

Rosenbaum P 2007 A report: The definition and classification of cerebral palsy. Developmental Medicine and Child Neurology S:1098-14

Tseng SH, Chen HC, Tam KW 2012 Systematic review and meta-analysis of the effect of equine assisted activities and therapies on gross motor outcome in children with cerebral palsy. Disability and Rehabilitation Early Online: 1-11

SniderL,Korner-BitenskyN,KammannC, WarnerS, Saleh M 2007 Horseback riding as therapy for children with cerebral palsy. Physical \& Occupational Therapy in Pediatrics 27(2): 5-23

Sterba, J.A. 2007. Does horseback riding therapy or therapist-directed hippotherapy rehabilitate children with cerebral palsy? Developmental Medicine \& Child Neurology, 49(1): 68-73.

Sussman, M.D. \& Aiona, M.D. 2004. Treatment of spastic diplegia in patients with cerebral palsy. Journal of Pediatric Orthopaedics B, 13(2): S1-S12.

Whalen CN, Case-Smith J 2011 Therapeutic effects of horseback riding therapy on gross motor function in children with cerebral palsy: A systematic review. Physical \& Occupational Therapy in Pediatrics [Online] Available: http:// www.ncbi.nlm.nih.gov/pubmed/22122355 [2012, May 12]

Three Gaits, Inc., Therapeutic Horsemanship Centre, Stoughton, Wisconsin. Available at http:// www.3gaits.org/ (accessed 08 March 2013) 